\title{
Some peculiarities of the mechanism of irreversible photostructural transformations in thin As-S-Se layers
}

\author{
A.V. Stronski \\ Institute of Semiconductor Physics NAS Ukraine, Prospect Nauki 45, 03028, Kiev, Ukraine
}

\begin{abstract}
The present paper is concerned with the peculiarities of the mechanism of irreversible photostructural transformations in As-S-Se layers. The starting point of the irreversible photostructural transformations is the state which is determined by the conditions of the fabrication of the As-S-Se layers. It is thermodynamically unstable and differs from the structure of the glasses or annealed films. Their state is the final one for the structure of asprepared layers changing under the influence of external factors. For the considered As-S-Se compositions the correlation of the composition dependencies for dispersion energy and optical dielectric constant of the exposed or annealed layers and structurally-dependent parameters of As-S-Se glasses: glass transition temperature $T_{g}$, relaxation enthalpy $\Delta H$, heat capacity $C_{p}$, mole volume, compactness, is characteristic. Irreversible photostructural transformations are characterized by the absence of the essential influence of the diffusion processes. This is connected with the close proximity and high concentration of the non-stoichiometric molecular fragments which contain homopolar (As-As, S-S (Se-Se)) bonds. The switching of the bonds with the decrease of the homopolar ones and various defects is energetically favorable. The consideration of the evolution of the number of such fragments (number of homopolar bonds) as a result of polymeric processes that take place due to the exposure influence and change the local structure of the amorphous layers towards that inherent for the glass, gives the exponential decay of their number with the increasing exposure. This is supported by the exponential decay of Raman spectra bands intensity with the exposure which correspond to the presence of molecular fragments containing homopolar bonds.
\end{abstract}

Keywords: chalcogenide vitreous semiconductors, As-S-Se layers, mechanism of photostructural transformations.

Paper received 28.12.00; revised manuscript received 14.02.01; accepted for publication 16.02.01.

\section{Introduction.}

Presence of various photoinduced effects (photopolymerization, photocrystallization, photodecomposition, photoinduced anisothropy, etc.) is characteristic for chalcogenide vitreous semiconductors (ChVS). This is connected with the several reasons: valence electrons in ChVS can be easily optically excited, with the strong electronphonon interaction in the flexible structural networks of ChVS, numerous metastable structures due to non-crystalline nature of ChVS. The structure of thermally evaporated ChVS films differs from that characteristic to massive ChVS glasses. Under the influence of external factors (exposure or annealing) due to polymerization processes caused by them, the structure of thermally evaporated ChVS films tends to that of glasses. The understanding of the nature of the photostructural transformations (which, despite the availability of several proposed models, remained in many aspects unclarified) during last decades attracted attention of many investigation groups. Photoinduced phenomena in ChVS can be classified according to their reversibility or irreversibility, anisotropy or isotropy, number of atomic sites involved in the observed photoinduced phenomena.

In this paper we will consider peculiarities of the mechanism of the irreversible photopolymeric processes, which involve large numbers of atomic sites and lead to the more stable atomic structures and also to the essential changes of such physico-chemical properties as density, hardness, elasticity, optical properties and chemical solubility in some etching solutions. We will not discuss possible mechanisms of the different reversible photoinduced phenomena, which lead to the substantially smaller changes, they are discussed in reviews [1-7]. Mechanisms of photoinduced anisotropy are discussed in [5-7]. 


\section{Results and discussion}

To the problem of the ascertaining mechanism of the irreversible photopolymerization processes substantially lesser attention was devoted after the difference of the structure of the as-evaporated ChVS films was established, and that their structure becomes nearer to the structure of the massive glasses under the actions of the external factors (exposure or annealing). But they represent considerable interest in many practical applications (inorganic resists in microlithography, registering media for information storage, holography).

At the present stage of investigations, physical properties of ChVS are analyzed within the frames of topological theories of the structure of ChVS glasses the actively developed during last decade. The base of the theoretical representations about glass structure is the continuous network model (CNM) according to Zakhariansen [8]. CNM model is based on two postulates: presence of the continuous spatial network and energy similarity of the glass and crystal. The structure of the glass is determined mainly by the short-range order, the interconnection of the main structural units is of second priority. At present the CNM model is mainly used as the model of an ideal glass structure, which, similar to an ideal crystal, in pure form is never realized. In recent yyears the factors (structural, chemical bonding, thermodynamical) that influence glass-forming ability were intensively investigated. In the microscopic theory of the glass formation for the covalent solid state bodies it was suggested that the valence forces such as bond-stretching (a) and bond-bending (b) forces can serve as the atomic limitations during formation of the covalent networks [9-12]. Moreover, a-forces are approximately 3 times (or more) stronger than b-forces. It was suggested that the glass formation will be optimized (the maximal mechanical stability of the glass will be achieved), when the number of limitations per atom will be equal to the dimensionality of the space $N_{d}=3$. For the three-dimensional networks in which the atom has coordination number $m \geq 2$, the number of $a-a n d ~ b$ limitations is given

$$
\begin{aligned}
& n_{\alpha}=m / 2 \\
& n_{\beta}=2 m-3
\end{aligned}
$$

The formation of the rigid structure occurs when

$n_{\alpha}+n_{\beta}=3$

or mean coordination number

$$
\tilde{m}=2.40
$$

At such critical $\tilde{m}$ value the formation of the infinite rigid cluster occurs (the threshold of the rigidity percolation). The condition of the maximal chemical stability «chemical ordering» which correspond to the mutual bonds saturation of the heterogenous atoms in the shortrange order is realized at $x=0.40$ for the compositions $\mathrm{As}_{\mathrm{x}}(\mathrm{S}, \mathrm{Se})_{1-\mathrm{x}}$ with $\tilde{m}=2.40$. Here it is necessary to note that for the same values of the coordination number and the respective compositions the presence of the crystalline compounds orpiment $\mathrm{c}-\mathrm{As}_{2} \mathrm{~S}_{3}$ which is isomorphic to c- $\mathrm{As}_{2} \mathrm{Se}_{3}$ is characteristic [13-14]. For such compositions the coincidence of the chemical and mechanical thresholds is characteristic.

Recently, the substantial progress was achieved in understanding the kinetic, thermal and structural factors which influence glass forming temperature $T_{g}$. Developed in [15] stochastic agglomeration theory provides possibility of the analysis of the $T_{g}$ compositional changes, which are characterize the changes in the glass connectivity. If the As additive in the $\mathrm{S}(\mathrm{Se})$ glass exists only in the three-coordinated (pyramidal units) configuration, then the $T_{g}$ dependence on the arsenic content $\mathrm{x}$ has the form:

$d T_{g} / d x=T_{0} / \ln (3 / 2)$

where $T_{0}$ - glass forming temperature of $\mathrm{Se}(\mathrm{S})$.

But for the glasses from the $\mathrm{As}_{\mathrm{x}} \mathrm{Se}_{1-\mathrm{x}}$ system experimentally obtained value $d T_{g} / d x$ was found to be equal 7.7 C/at.\% [16-17], and obtained from the expression (3)4.1 C/at.\%. This was explained by the presence in the glass of the certain number of the fourfold coordinated $\mathrm{As}^{5+}$ in the quasi-tetrahedral local configuration with 3 onefold Se atoms bounded to the arsenic and one twofold bounded $\mathrm{Se}$ in addition to the $\mathrm{As}^{3+}$ in the pyramidal configurations in these glasses. In this case, the Eq. (3) takes more general form

$d T_{g} / d x=T_{0} / \ln (3 / 2)(1-\eta)$

In Eq. (4) $\eta$ is the ratio of the $\mathrm{As}^{5+}$ to the $\mathrm{As}^{3+}$ cation in the glass network. To match experimentally observed slope in the dependence (4) the values of $\eta$ must be close to 0.3 . That is, the given results show that the traditional molecular structure of such glasses can be incomplete and, in particular, As in addition to the pyramidal configuration $\left(\mathrm{As}^{3+}\right)$ can be also in the quasi-tetrahedral configuration $\left(\mathrm{As}^{5+}\right)$ in the glasses with the high content $(x<0.4)$ of selenium (sulfur). Using results of Raman spectra investigations in $\mathrm{As}_{\mathrm{x}} \mathrm{S}_{1-\mathrm{x}}$ glasses, the existence of fourfold coordinated arsenic in such glasses was assumed [18-19]. The presence of the different structural units in the considered compositional region is supported by the data of Raman spectra investigations for the glasses and films from this compositional region. Besides, the presence of the wide weak band (maximum in the $460-480 \mathrm{~cm}^{-1}$ region) in the Raman spectra of the $\mathrm{As}_{40} \mathrm{Se}_{60}$ glasses [20-21], can also correspond to the presence of the fourfold coordinated arsenic [22]. The theoretical calculations shows the presence of the two rigidity thresholds $\left(x_{C}(1)=0.29\right.$ and $\left.x_{C}(2)=0.37\right)$ in binary glasses of $\mathrm{As}_{\mathrm{x}} \mathrm{Se}_{1-\mathrm{x}}$ composition $\left(\right.$ or $\left.A s_{\mathrm{x}} \mathrm{S}_{1-\mathrm{x}}\right)$ [17]. If only threefold coordinated arsenic is present, the rigidity threshold is expected at $x_{C} \sim 0.40$, which correspond to the mean coordination number $\tilde{m}=$ 2.40 [17]. The four regions of the arsenic concentration can be separated during description of the structural peculiarities of the $\mathrm{As}_{\mathrm{X}} \mathrm{S}(\mathrm{Se})_{1-\mathrm{x}}$ glasses. The glasses with the 


\section{A.V. Stronski: Some peculiarities of the mechanism of irreversible photostructural ...}

low connectivity $(x<0.15)$ are well described within the frames of the random network model or stochastic model (4). At larger values the composition dependencies of the glass forming temperature deviate from the dependence (4), because the configuration entropy of the network become lower, when the separate chemical configurations became preferable. This reflects the non-stochastic nature of the rigid configurations formation with the increasing $x$. Between both rigidity thresholds $x_{C}(1)=0.29$ and $\left.x_{C}(2)=0.37\right)$ in the binary As-Se glasses the existence of the intermediate phase is predicted (for which the absence of the internal stresses is characteristic) with the rigid and soft regions for which the chemistry and package of the network is important [17]. At $x_{C}>0.37$ the transition to a rigid stressed phase occurs.

The presented in Fig. 1 composition dependencies of the mole volumes for the glasses of $\mathrm{As}_{100-\mathrm{x}} \mathrm{S}_{\mathrm{x}}$ and $\mathrm{As}_{40} \mathrm{~S}_{60-\mathrm{x}} \mathrm{Se}_{\mathrm{x}}$ compositions are similar to the presented in [14]. The mole volume is determined by the relation

$$
V=\left(\sum x_{i} M_{i}\right) / \rho
$$

where $M_{i}$ stands for molecular masses of the certain structural units or atomic masses of components, $x_{i}$ is their respective content, $\rho$ is measured glass density.

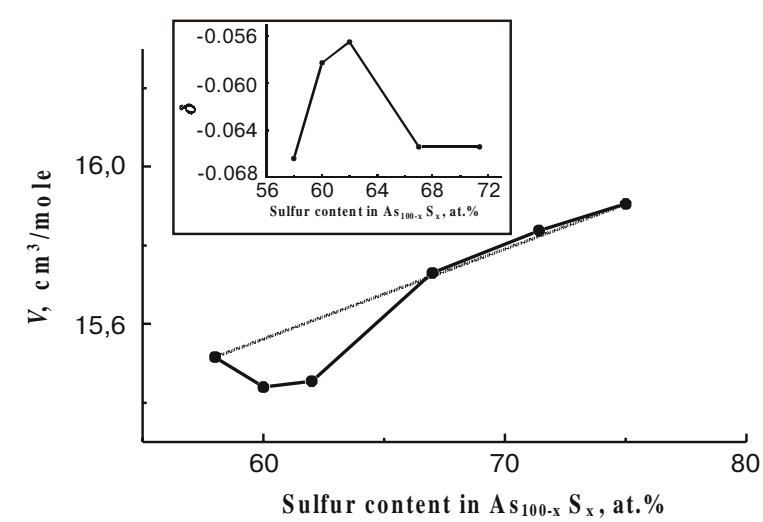

a)

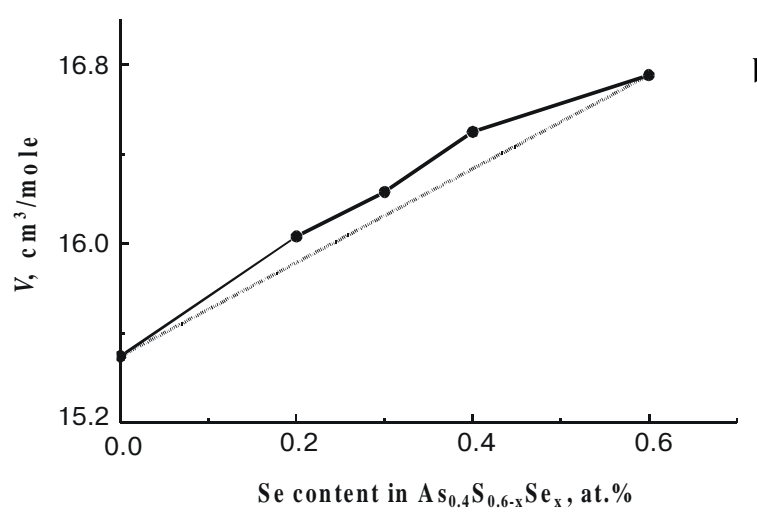

Fig. 1. a - Dependence of the mole volume $V$ of $\mathrm{As}_{100-\mathrm{x}} \mathrm{S}_{\mathrm{x}}$ glasses on the sulphur content. Insert - dependence of the compactness $\delta$ of $\mathrm{As}_{100-\mathrm{x}} \mathrm{S}_{\mathrm{x}}$ glasses on the sulphur content; $\mathrm{b}$ - Dependence of the mole volume $V$ of $\mathrm{As}_{40} \mathrm{~S}_{60-\mathrm{x}} \mathrm{Se}_{\mathrm{x}}$ glasses on the selenium content.
When arsenic is introduced into sulfur, the structure of $\mathrm{As}_{100-\mathrm{x}} \mathrm{S}_{\mathrm{x}}$ glasses gradually changes from the chain structure to the network one with the formation of the $\mathrm{AsS}_{3 / 2}$ structural units, etc., therefore mean mole volume is decreased. Deviation from the linear dependence (which is observed for $x>75 \%$ [14]) is characteristic for the compositions with $x$ ranging from 25 up to $42 \mathrm{at} . \%$. At $x=0.4$ minimum is observed (for As-Se system minimum $V$ at $x=0.4$ is also present [14]). The anticorrelation is characteristic for the composition dependencies of the glass mole volumes on the composition cross-section $\mathrm{As}_{100-\mathrm{x}} \mathrm{S}_{\mathrm{x}}$ (Fig. 1a) and values of irreversible photostructural changes of $\mathrm{As}_{100-\mathrm{x}} \mathrm{S}_{\mathrm{x}}$ layers ([23], see Fig.2), namely, minimum of mole volume composition dependence $(x \sim 0.4)$ correspond to the maximum of the composition dependence of photostructural changes. Deviation of mole volume composition dependence for $\mathrm{As}_{40} \mathrm{~S}_{60-\mathrm{x}} \mathrm{Se}_{\mathrm{x}}$ glasses (Fig. 1b) from the linear one we consider in connection with the deviation in the substitution of sulfur by selenium from the statistical one. Due to such behavior the number of sulfur deficient (in comparison to the $\mathrm{AsS}_{3 / 2}$ ) structural units, which are characteristic for $\mathrm{As}_{4} \mathrm{~S}_{4}$ is increased. This leads to the presence in the $\mathrm{As}_{40} \mathrm{~S}_{60-\mathrm{x}} \mathrm{Se}_{\mathrm{x}}$ glass structure of the increased quantities of $\mathrm{Se}_{n}$ chains for the intermediate compositions and, consequently, to

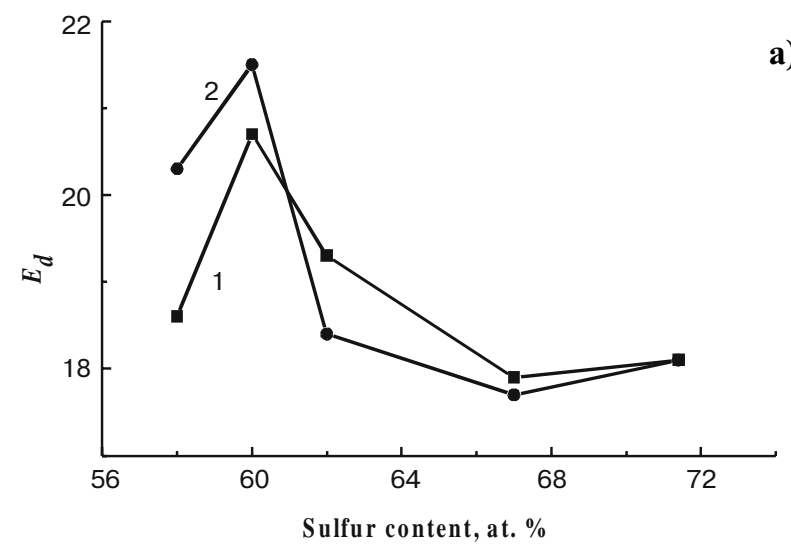

a)

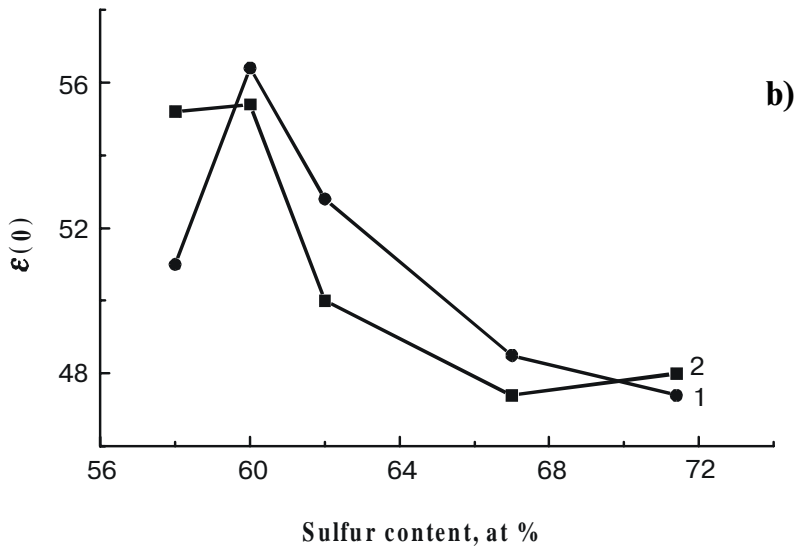

Fig. 2. a - Dependence of the dispersion energy $E_{d}$ of $\mathrm{As}_{100-\mathrm{x}} \mathrm{S}_{\mathrm{x}}$ layers on the sulphur content: 1 - annealed, 2 - exposed layers [23]. $\mathrm{b}$ - Dependence of the optical dielectric constant of $\mathrm{As}_{100-\mathrm{x}} \mathrm{S}_{\mathrm{x}}$ layers on the sulphur content: 1 - annealed, 2 - exposed layers [23]. 
the slight deviation (small increase due to the presence of chain structure) from the linear one of the mole volume composition dependence. Deviation of mole volume composition dependence for $\mathrm{As}_{40} \mathrm{~S}_{60-\mathrm{x}} \mathrm{Se}_{\mathrm{x}}$ glasses correlates with the little features on the composition dependencies of dispersion energy and optical dielectric constant for exposed or annealed films $\mathrm{As}_{40} \mathrm{~S}_{60-\mathrm{x}} \mathrm{Se}_{\mathrm{x}}$ (see Fig. 3) [24].

In some works [25-26] it was supposed that besides bond switching, the presence of the free volume is also influencing the magnitude of the photostructural changes. The compactness $\delta$, which is supposed to be more sensitive to the structure of the glass network, is calculated according to the formula

$$
\delta=\frac{\sum_{i} \frac{x_{i} A_{i}}{\rho_{i}}-\sum_{i} \frac{x_{i} A_{i}}{\rho}}{\sum_{i} \frac{x_{i} A_{i}}{\rho}}
$$

where $x_{i}$ is an atomic fraction, $A_{i}$-atomic weight, $\rho_{i}-$ density of the glass $i$ component, $\rho$-measured glass density.
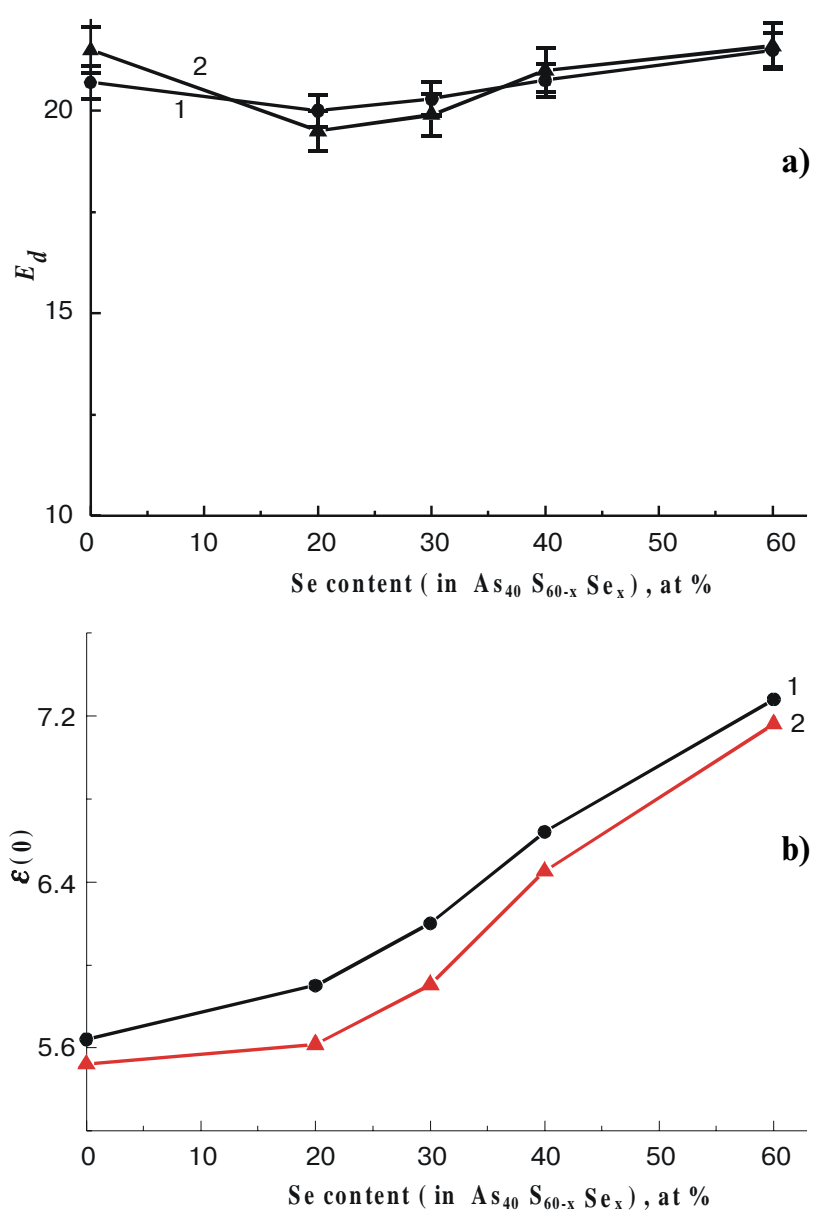

Fig. 3. a - Dependence of the dispersion energy $E_{d}$ of $\mathrm{As}_{40} \mathrm{~S}_{60-\mathrm{x}} \mathrm{Se}_{\mathrm{x}}$ layers on the selenium content: 1 - annealed, 2 - exposed layers [24]. $\mathrm{b}$ - dependence of the optical dielectric constant of $\mathrm{As}_{40} \mathrm{~S}_{60-\mathrm{x}} \mathrm{Se}_{\mathrm{x}}$ layers on the selenium content: 1 - annealed, 2 - exposed layers [24].
Indeed, calculated compactness (using the data from Fig. 1, see insert in Fig.1a) for the glasses of $A s_{100-x} S_{x}$ compositions gives clear feature (maximum) in the region $60-62$ aт. $\%$ of sulfur content (that is in the region of the stoichiometric composition $\mathrm{As}_{40} \mathrm{~S}_{60}$ ), which correlates with the maximum of photostructural changes (see Fig. 2). But here it is necessary to note that the compactness, by its definition, mainly reflects the structural peculiarities of the final stage of photoinduced transformations.

The magnitude of the photostructural changes in As-S-Se films depends on several factors, and among them, let us note the degree of their disorder (which can be varied by means of changing preparation conditions), the degree of their structure deviation from the structure of the glass structure network (structure of annealed or exposed film). It is noteworthy that for $\mathrm{As}_{100-\mathrm{x}} \mathrm{S}_{\mathrm{x}}$ compositions at the same $x$ values, when this peculiarities are observed $(x \sim 0.286$ and $x \sim 0.4)$, the presence of the compounds which exist in crystalline form is characteristic: $\mathrm{As}_{2} \mathrm{~S}_{3}$ and $\mathrm{As}_{2} \mathrm{~S}_{5}$ [13-14, 27]. For more accurate correspondence of the experimental data and predicted by the topological theories composition behavior, it is necessary to carry out the investigations along the given compositional cross-sections with sufficiently less step for $x$. Here it is necessary to emphasize that the peculiarities of the composition dependencies (observed near $x \sim 0.286$ and $x \sim 0.4$ ) of the dispersion energy, optical dielectric constant, and also their changes that characterize the magnitude of the photostructural transformations in the films of $\mathrm{As}_{\mathrm{x}} \mathrm{S}_{1-\mathrm{x}}$ composition, and such structurally-dependent parameters as glass forming temperature $T_{g}$, relaxation enthalpy $\Delta H$, heat capacity $C_{p}$ for the glasses from $\mathrm{As}_{\mathrm{x}} \mathrm{S}_{1-\mathrm{x}}$ line [18-19] correlate with the positions predicted by the theory for the two rigidity thresholds (Fig. 4). For glasses from $\mathrm{As}_{\mathrm{x}} \mathrm{Se}_{1-\mathrm{x}}$ compositional cross-section [16], also characteristic is the correlation of the peculiarities of $T_{g}, \Delta H, C_{p}, \Delta C_{p}$ compositional dependencies with

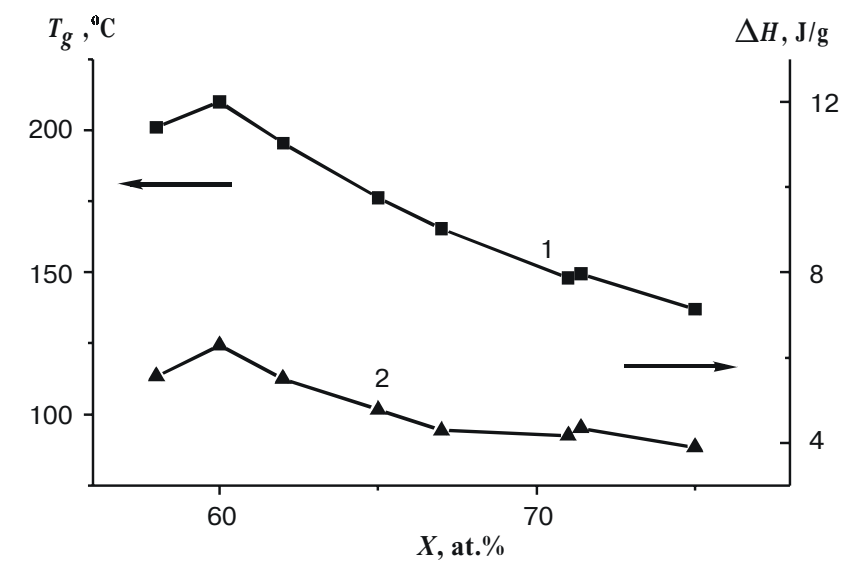

Fig. 4. Dependence of the glass-forming temperature $T_{g}$ (1) and relaxation enthalpy $\Delta H(2)$ of $\mathrm{As}_{100-\mathrm{x}} \mathrm{S}_{\mathrm{x}}$ glasses on the sulfur content [18-19]. 


\section{A.V. Stronski: Some peculiarities of the mechanism of irreversible photostructural ...}

the position of the predicted by the topological theory two rigidity thresholds. Peculiarity of the composition dependence of mole volume for the $\mathrm{As}_{40} \mathrm{~S}_{60-\mathrm{x}} \mathrm{Se}_{\mathrm{x}}$ glasses (Fig. 1b) for the intermediate compositions corresponds to the weak peculiarity for the composition dependencies of dispersion energy and optical dielectric constant for $\mathrm{As}_{40} \mathrm{~S}_{60-\mathrm{x}} \mathrm{Se}_{\mathrm{x}}$ layers and peculiarities for compositional dependencies of $T_{g}, \Delta H, C_{p}$ for $\mathrm{As}_{40} \mathrm{~S}_{60-\mathrm{x}} \mathrm{Se}_{\mathrm{x}}$ glasses [24] (see Fig. 5). Such correlation of peculiarities should be expected, because the change of the structure with the composition for glasses must be reflected in compositional dependencies of the such structurally dependent parameters as $T_{g}, \Delta H, C_{p}$. In their turn, the composition dependencies of dispersion energy and optical dielectric constant for As-S-Se layers (annealed or exposed, which is the similar same to the structure of the glasses) are influenced by the structural changes with the composition.

Thus, the conclusion can be made, that the peculiarities of the composition dependencies of the photostructural transformations in As-S-Se are determined by the structural properties of the glasses and films as well as energetic preference of the structural relaxation of the initial
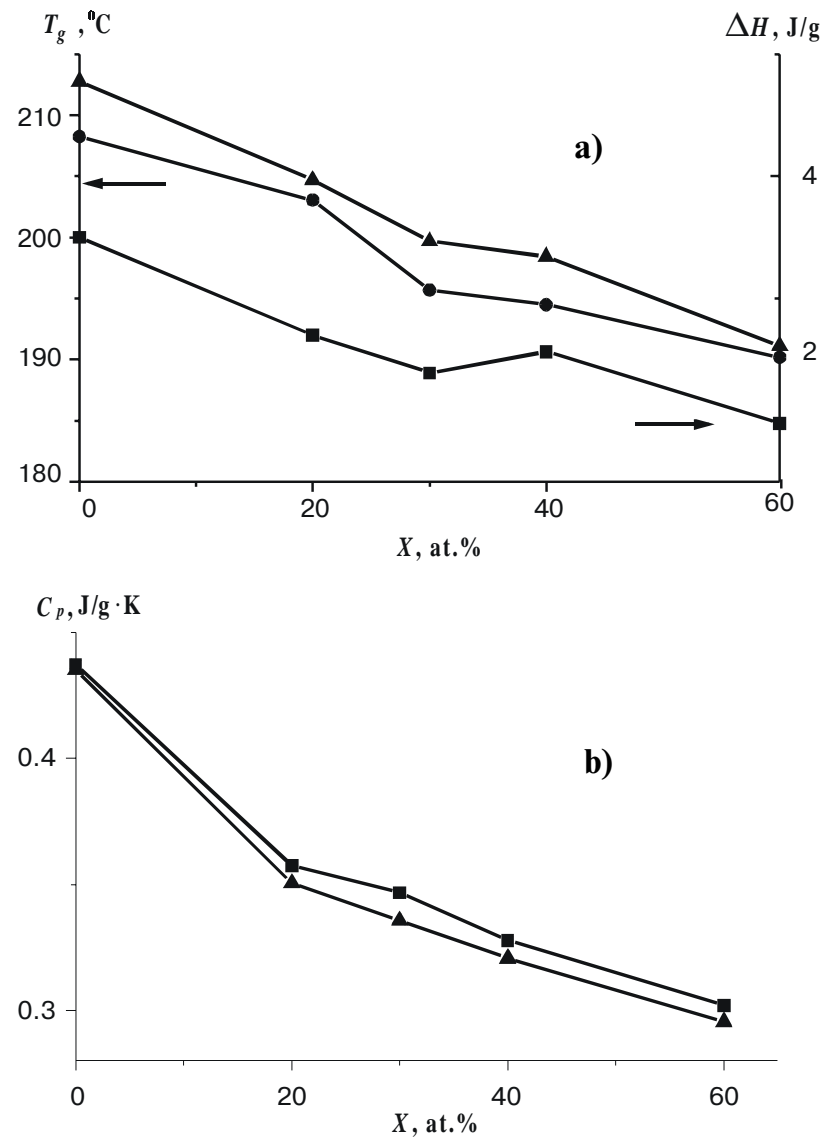

Fig. 5. a - Dependence of the glass-forming temperature $T_{g}$ (1) and relaxation enthalpy $\Delta H$ (2) of $\mathrm{As}_{40} \mathrm{~S}_{60-\mathrm{x}} \mathrm{Se}_{\mathrm{x}}$ glasses on the selenium content [24]; $\boldsymbol{-}, \boldsymbol{\Delta}$ refer to heating and cooling scans. $\mathrm{b}-$ Dependence of the heat capacity $C_{p}$ of of $\mathrm{As}_{40} \mathrm{~S}_{60-\mathrm{x}} \mathrm{Se}_{\mathrm{x}}$ glasses on the selenium content [24] $\boldsymbol{\sim}, \boldsymbol{\Delta}$ refer to heating and cooling scans. fresh-evaporated films towards that characteristic for the annealed films (massive glasses).

As was said above the photostructural changes during irreversible photopolymerization processes involve density of atomic sites on the level from several atomic percents up to thirty percents [28-30]. The presence of molecular fragments containing homopolar As-As and $\mathrm{S}(\mathrm{Se})-\mathrm{S}(\mathrm{Se})$ bonds on the level 10-35 ат.\% in as-evaporated As-S(Se) films [28-30] means that the pairs of such molecular fragments with As-As and $\mathrm{S}(\mathrm{Se})-\mathrm{S}(\mathrm{Se})$ bonds due to their statistical distribution in the film are in close proximity to each other. The presence of such molecular fragments in the films is connected with the molecular composition of the vapor which is partially frozen during condensation on the substrate. Under the influence of external factors (exposure by light or thermal annealing) interaction takes place between As-As and S(Se)-S(Se) containing homopolar fragments which leads to the switching of the bonds, with the increase of the $\mathrm{AsS}(\mathrm{Se})_{3 / 2}$ stoichiometric structural units quantity.

The magnitude of such structural changes depends on the difference of the micro-heterogenic structure for the initial state of as-evaporated films from the structure characteristic to the films after the exposure or annealing. This difference (in the structure, content of the non-stoichiometric molecular fragments, deficit of density in comparison to those of massive glasses and annealed films) depends on the peculiarities of the film deposition process. Changes in values of single-oscillator model parameters (dispersion energy and optical dielectric constant) indicate the change of the short-range order, which is also supported by evolution of the Raman spectra of the films under the exposure or annealing [21, 24, 31-34] (Fig. 6 [33]). The change of the X-rays diffraction pattern for the films in the region of the first maximum after exposure or annealing also indicates the change of the

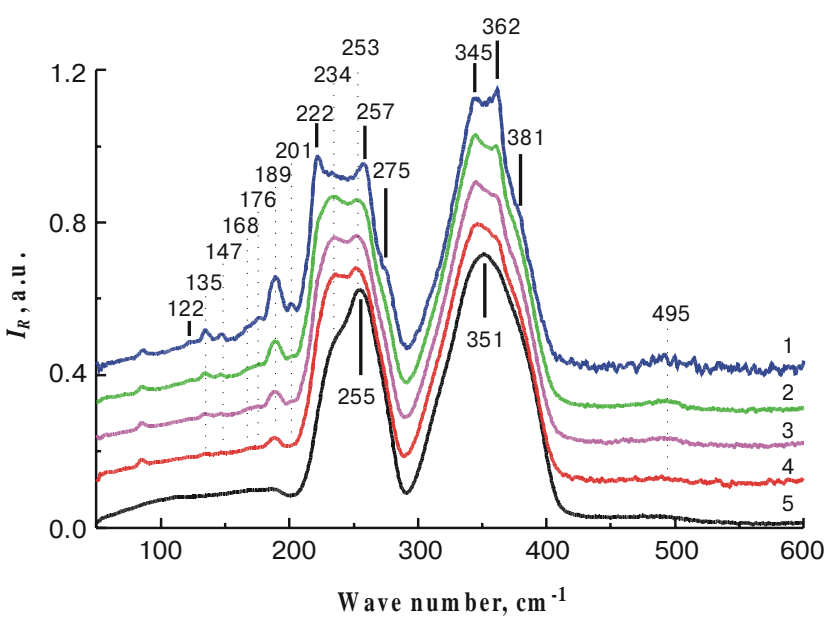

Fig. 6. Raman spectra of $\mathrm{As}_{40} \mathrm{~S}_{40} \mathrm{Se}_{20}$ [33]: 1 - as-evaporated layer, 2,3,4 - exposed (halogen lamp, $I=20 \mathrm{~mW} / \mathrm{cm}^{2}$, IR cutoff filter) $20,40,80 \mathrm{~min}$ of exposure respectively; 5 - bulk glass. Band areas have been reduced by the Shuker-Gammon method, normalized by an area and shifted. 
coordination of the nearest neighborhood. The disappearance of the first sharp diffraction peak after exposure or annealing [3] which is connected with the presence of the intermediate volume (voids) between main pyramidal structural units, is explained by the decrease of the volume of such voids, increase of the film density after exposure or annealing. The change of the short-range order in $\mathrm{As}_{2} \mathrm{~S}_{3}$ and $\mathrm{As}_{3} \mathrm{Se}_{3}$ films as a result of the exposure or annealing is supported by the results of the EXAFS investigations [34]. First coordination sphere according to [34] in the as-evaporated $\mathrm{As}_{2} \mathrm{~S}_{3}$ film contain $2.4 \mathrm{~S}$ atoms at $2.26 \AA$ distance and 0.6 As atoms at $2.54 \AA$ distance. After annealing the first coordination sphere is changed up to $2.8 \mathrm{~S}$ atoms at $2.27 \AA$ distance and 0.2 As atoms at $2.56 \AA$ distance. Similar changes are observed also for $\mathrm{As}_{2} \mathrm{Se}_{3}$ films. It means that the substantial part of the «wrong» homopolar bonds is eliminated under annealing. The annealed or exposed films and massive glasses are very similar in the short-range order structure. This is supported by the consideration of the Raman spectra evolution of the As-S-Se films under exposure or annealing $[21,24,31-33]$. The possible variants of the bond configurations and their switching under external influences are considered in detail elsewhere [1, 3-4, 7, 35-36].

As we already noted, the irreversible structural changes (photopolymerization) start from the initial states which are determined by the films preparation conditions and are different from the glass structure or from the structure of the annealed film. In the considered case there is no significant influence of the diffusion processes, because due to the high concentration (as was said above up to 30 at.\%) the pairs (enriched by As and $\mathrm{S}(\mathrm{Se})$ ) of homopolar fragments are in the nearest proximity to each other (within the limits of the short-range order). Also it was noted the specific feature of the amorphous chalcogenides is the rapid localization of the photoexcited carriers within the region of the order of several angstroems $[1,4]$. Bonds switching with the decrease of the homopolar bonds number are favorable thermodynamically. Thus, the change of the number $N_{H}$ of placed in nearest proximity each other As-As and $\mathrm{S}(\mathrm{Se})-\mathrm{S}(\mathrm{Se})$ homopolar fragments in the initial as-evaporated film and, respectively, the number of the homopolar bonds under exposure can be written as [23, 33]:

$$
d N_{H} / d t=-C \alpha I N_{H}
$$

where $\alpha$ is an absorption coefficient, $I$ - light intensity, $C$ - constant.

The solution of the equation (7) is written in the form

$$
N_{H}=N_{H_{0}} \exp (-C \alpha H)
$$

where $N_{H_{0}}$ is the initial number of the molecular fragments which contain homopolar As-As and S(Se)-S(Se) bonds (that is the number of the respective homopolar bonds) which are in the nearest proximity to each other in the initial as-evaporated film, $H=I \cdot t-$ exposure value.
Such consideration is supported by the exponential decay behavior for the intensity dependencies on exposure of the Raman bands which correspond to the presence of the molecular fragments containing homopolar bonds in the Raman spectra of the $\mathrm{As}_{100-\mathrm{x}} \mathrm{S}_{\mathrm{x}}$ and $\mathrm{As}_{40} \mathrm{~S}_{60-\mathrm{x}} \mathrm{Se}_{\mathrm{x}}$ films $[23,33]$. Besides, the experimentally observed exponential decay behavior for the etch rate $V_{e}$ dependencies on exposure for the As-S-Se layers [23-24, 33] also can be considered as the support of the presented above consideration, if the mechanism of selective etching is connected with the attack on the homopolar fragments. Here it is necessary to note that etching can proceed in several stages [37], different mechanisms are possible, and thus the interconnection of the $V_{e}$ and $N_{H_{0}}$ can be expressed in essentially more complex way.

\section{Conclusions}

Thus, the conclusion can be made, that the peculiarities of the composition dependencies of the photostructural transformations in As-S-Se are determined by the structural properties of the glasses and films (the final state to which the structure of layers tends under the influence of the external factors) and energetic preference of the structural relaxation of the thermodynamically unstable initial as-evaporated films towards the structures characteristic for the annealed films (massive glasses).

\section{References}

1. H. Fritzche, The origin of the reversible and non-reversible photo-structural transformations in chalcogenide glasses // Philosophical Magazine B., 68 (4), pp. $561-572$ (1993).

2. P.J.S. Ewen and A.E. Owen, Photoinduced changes in chalcogenide glasses and their applications. In. High-Performance Glasses, Eds. M.Carble and J.M.Parker, «Blackie», Glasgow and London, pp. 287-337 (1992).

3. S.R. Elliott, A unified model for reversible photostructural effects in chalcogenide glasses // J.Non-Cryst.Sol., 81 (1-2), pp. 71-98 (1986).

4. H. Fritzsche, Towards understanding the photo-induced changes in chalcogenide glasses // FTP (Semiconductors), 32 (8), pp. 952-957 (1998).

5. V.K. Malinovsky, About the mechanism of photo-transformations in the optical recording registering media // Autometry, №1, pp. 25-49 (1985).

6. V.K. Tikhomirov, Photoinduced effects in undoped and rareearth doped chalcogenide glasses: review // J.Non-Cryst. Sol., 256\&257, pp. 328-336 (1999).

7. K. Tanaka, Photoinduced structural changes in amorphous semiconductors // FTP (Semiconductors), 32 (8), pp.964969 (1998).

8. C.A. Dembovsky and E.A. Chechetkina, Glass formation, Nauka, Moscow, 1990, 278p. (in Russian).

9. J.C.Phillips, Topology of covalent non-crystalline solids II: medium range order in chalcogenide alloys and a-Si $(\mathrm{Ge})$ / / J.Non-Cryst.Sol., 43 (1), pp.37-77 (1981).

10. J.C.Phillips, The physics of glass // Physics Today, February, pp.27-33 (1982).

11. J.C.Phillips. and M.F. Thorpe, Constraint theory, vector percolation and glass formation // Solid State Communs., 53 (8), pp.699-702 (1985). 


\section{A.V. Stronski: Some peculiarities of the mechanism of irreversible photostructural ...}

12. M.F. Thorpe and D.J. Jacobs, The structure and rigidity of network glasses. In: Insulating and Semiconducting Glasses (Series on directions in condensed matter physics, v.17), Editor P.Boolchand, World Scientific, Singapore, 2000, pp. 95-146.

13. S.V.Svechnikov, V.V.Khiminets, N.I. Dovgoshey, Complex non-crystalline chalcogenides and chalcohalogenides and their applications in optoelectronics Kiev: Naukova Dumka, 1992, 296p. (in Russian).

14. A. Feltz, Amorphous and vitreous inorganic solid states. Mir, Moscow 1986, 556p. (in Russian).

15. G.G.Naumis, R. Kerner Stochastic matrix description of glass transition in ternary chalcogenide systems // J.Non-Cryst. Solids, 231, pp. 111-119 (1998).

16. T. Wagner and S.O.Kasap, Glass transformation, heat capacity and structure of $\mathrm{As}_{\mathrm{x}} \mathrm{Se}_{1-\mathrm{x}}$ glasses studied by modulated temperature differential scanning calorimetry experiments // Phil. Mag. B, 74, (6) pp.667-680 (1996).

17. Insulating and semiconducting glasses (Series on directions in condensed matter physics, v.17), Editor P.Boolchand, World Scientific, Singapore, 2000.-882p.

18. T.Wagner, S.O.Kasap, M.Vlček, A.Sklenař, A.Stronski, The structure of $\mathrm{As}_{\mathrm{x}} \mathrm{S}_{100-\mathrm{x}}$ glasses studied by temperature modulated differential scanning calorimetry and Raman spectroscopy // J.Non-Cryst.Solids, 227-230, Pt.II, pp.752-756 (1998)

19. T.Wagner, S.O.Kasap, M.Vlček, A.Sklenař, A.Stronski, Temperature-modulated differential scanning calorimetry and Raman spectroscopy studies of $\mathrm{As}_{\mathrm{x}} \mathrm{S}_{100-\mathrm{x}}$ glasses // Journal of Material Science, 33 (I.23) pp. 5581-5588 (1998).

20. M.Vlček, A.Stronski, A.Sklenař, S.A.Kostioukevitch, and P.F.Romanenko, Photoimaging properties and imaging technology on the base of $\mathrm{As}_{40} \mathrm{Se}_{60}$ thin layers // Proceedings of SPIE, 3450, pp. 125-132 (1998).

21. A.V.Stronski, M.Vlček, A.Sklenař, P.F.Romanenko, S.A. Kostyukevich, Lightsensitive properties of $\mathrm{As}_{40} \mathrm{Se}_{60}$ layers //Optoelectronics and semiconductor technique, I.34, pp. 65-71 (1999).

22. P.Boolchand, private communication.

23. A.Stronski, M.Vlček, A. Sklenař, Photoinduced structural changes in $\mathrm{As}_{100-\mathrm{x}} \mathrm{S}_{\mathrm{x}}$ layers // Semiconductor Physics, Quantum Electronics \& Optoelectronics, 3 (3), pp. 394-399 (2000).

24. M.Vlček, A.Stronski, A.Sklenař, T.Wagner and S.O. Kasap Structure and Imaging properties of $\mathrm{As}_{40} \mathrm{~S}_{60-\mathrm{x}} \mathrm{Se}_{\mathrm{x}}$ layers as a function of their composition // J.Non-Cryst.Solids, 266-269 (I.1-3) pp. 964-968 (2000).
25. M.Vlček, M. Frumar and A.Vidourek, Photoinduced effects in Ge-Sb-S glasses and amorphous layers // J.Non-Cryst. Solids, 90 (1-3), pp. 513-516 (1987).

26. G. Saffarini, Compositional trends of the compactness in ternary chalcogenide glasses of the Ge-In-Se system // Physica $\boldsymbol{B}, \mathbf{2 5 3}$, pp. $52-55$ (1998).

27. Z.U. Borisova, Chalcogenide semiconductor glasses, L.: Leningrad University, 1983.-344p. (in Russian).

28. Amorphous semiconductors. Ed. M.Brodsky. Mir, Moscow 1982.- 416p. (in Russian).

29. F.Kosek, Z.Cimpl, J.Tulka, J.Chlebny, New analytic method for investigation of the distribution of bonds in As-S systems / / J.Non-Cryst.Solids, 90 (3), pp.401-404 (1987).

30. N.D. Aksenov, L.L. Makarov, S.B. Mamedov Chemical ordering in $\mathrm{As}_{2} \mathrm{Se}_{3}$ films under photostructural transformations. In.: Non-crystalline semiconductors-89, «Patent», Uzhgorod, 2, pp.192-194 (1989).

31. A.V.Stronski, M.Vlček, P.E.Shepeljavi, A.Sklenař, S.A. Kostyukevich, Image formation properties of $\mathrm{As}_{40} \mathrm{~S}_{20} \mathrm{Se}_{40}$ thin layers in application for gratings fabrication // Semiconductor Physics, Quantum Electronics \& Optoelectronics, 2 (1), pp.111-114 (1999).

32. A.V.Stronski, M.Vlček, A.I.Stetsun, A.Sklenař, P.E. Shepeljavi, Raman spectra of Ag- and Cu-photodoped $\mathrm{As}_{40} \mathrm{~S}_{60-\mathrm{x}} \mathrm{Se}_{\mathrm{x}}$ films // J.Non-Cryst.Solids, 270 (I.1-3), pp.129-136 (2000).

33. A.Stronski, M.Vlček, Imaging properties of $\mathrm{As}_{40} \mathrm{~S}_{40} \mathrm{Se}_{20}$ layers // Optoelectronics Review, 8, N3, pp.63-67, 2000.

34. R.J. Nemanich, G.A.N. Connel, T.M. Hayers, and R.A. Street, Thermally induced effects in evaporated chalcogenide films.I. Structure // Phys.Rev. B, 18 (12), pp. 6900-6914 (1978).

35. O.I. Shpotyuk, On the mechanism of reversible radiationstructural transformations in chalcogenide vitreous semiconductors // Journal of Applied Spectroscopy, 59 (5-6) pp.550553 (1993).

36. S.A.Dembovsky, S.A.Zyubin, F.V.Grigoriev, The modelling of the hypervalent configurations, valence alternation pairs, deformed structure and properties of the a-S and a-As $\mathrm{S}_{3} / /$ FTP (Physics and Technology of Semiconductors), 32 (8) pp. 944-951 (1998).

37. S.A.Zenkin, S.B.Mamedov, M.D.Mikhailov, E.Yu.Turkina and I.Yu.Yusupov Mechanism for interaction of amine solutions with monolithic glasses and amorphous films in the AsS system // Glass Physics and Chemistry, 23 (5) pp. 393-399 (1997) 